an allogeneic mixed-lymphocyte reaction (MLR). Lymphocyte proliferation (flow-cytometric measurement of CFSE) and cytokine production (multiplex bead array) were assessed at day 5 . The proportion of lymphocytes primed to produce IFN $\gamma$ was measured at day 7 (intracellular staining).

Results UPM-stimulation increased DC expression of the maturation marker CD83 ( $\mathrm{p}=0.0038)$ and chemokine receptor CCR7 ( $p=0.0018$ ). It had no effect on CD40 or MHC Class I expression. UPM-stimulation of DCs also significantly increased CD8 lymphocyte proliferation $(\mathrm{p}=0.020)$, and the production of IFN $\gamma$, TNF $\alpha$ and IL-13 by CD8 lymphocytes in MLR at day 5 (all $\mathrm{p}<0.05$; Table 1). The proportion of CD8 lymphocytes primed to produce IFN $\gamma$ was also increased by UPM-stimulation of DCs $(\mathrm{p}=0.034)$.

Conclusion No evidence of an impaired Tc1 response was seen with UPM-stimulated DCs, in contrast to our previous findings with CD4 T lymphocytes. This may be because CD8 lymphocytes are more primed to respond and produce cytokines at baseline. However, UPM-treatment of DCs did significantly increase DC expression of CCR7, which directs DCs to lymph nodes, and increased the priming of Tc1 and Tc2 responses in the absence of any other stimulation. Inhalation of UPM may give rise to pathological CD8 responses to otherwise innocuous novel antigens.

\section{S49 TELOMERE ATTRITION IN CIRCULATING WHITE BLOOD CELLS IN COPD RELATES TO LUNG FUNCTION AND OUTCOMES}

${ }^{1}$ Roberto A Rabinovich, ${ }^{1}$ Gourab Choudhury, ${ }^{1}$ Ramzi Lahkdar, ${ }^{1}$ Ellen M Drost, ${ }^{2}$ Liane McGlynn, ${ }^{1}$ Jing Bai, ${ }^{2}$ Paul G Shiels, ${ }^{3}$ Bruce E Miller, ${ }^{3}$ Ruth Tal-Singer, ${ }^{4}$ Alvar Agusti, ${ }^{1}$ William MacNee. 'Edinburgh Lung and the Environment Group Initiative (ELEGI), Centre for Inflammation and Research, Queens Medical Research Institute, Edinburgh, Edinburgh, UK; ${ }^{2}$ University of Glasgow, College of Medical, Veterinary and Life Sciences Institute of Cancer Sciences, Glasgow, UK; ${ }^{3}$ GlaxoSmithKline $R$ and D, King of Prussia, Pennsylvania, USA; ${ }^{4}$ Hospital Clinic, IDIBAPS, Universitat de Barcelona and CIBER Enfermedades Respiratorias, FISIB, Mallorca, Spain

\subsection{6/thoraxjnl-2014-206260.55}

Introduction Increasing evidence suggests accelerated ageing as a pathogenic mechanism in COPD.

Methods and results Telomere length in circulating WBC, a marker of biological ageing, was assessed in 200 ex-smoker COPD patients $\left(108\right.$ male, age $61.5 \pm 6.4$ years, $\mathrm{FEV}_{1} 45.6 \pm$ $17.1 \%$ predicted), 50 ex-smokers with normal lung function (27 male, age $59.9 \pm 7.3$ years, $\mathrm{FEV}_{1} 109.1 \pm 13.4 \%$ predicted) and 50 non-smoker healthy subjects $(27$ male, age $59.3 \pm 8.3$ years, $\mathrm{FEV}_{1} 113.2 \pm 13.1 \%$ predicted). TL was assessed by qPCR and expressed as relative $\mathrm{T} / \mathrm{S}$ ratio.

$\mathrm{TL}$ was shorter in COPD $(0.77 \pm 0.2$ relative $\mathrm{T} / \mathrm{S}$ ratio $)$ than in both ex-smokers $(0.83 \pm 0.2$ relative $\mathrm{T} / \mathrm{S}$ ratio $)$ and nonsmokers $(0.84 \pm 0.2$ relative $\mathrm{T} / \mathrm{S}$ ratio) $(\mathrm{p}<0.05)$. Furthermore TL correlated negatively with age ( $\mathrm{r}-0.17, \mathrm{p} 0.007)$, emphysema score ( $\mathrm{r}-0.217$, $\mathrm{p}$ 0.001), number of exacerbations in the previous year to inclusion in the study ( $\mathrm{r}-0.129, \mathrm{p} 0.04)$, number of hospitalisations over 3 years follow-up ( $\mathrm{r}-0.167, \mathrm{p}$ 0.004) and positively with $\mathrm{FEV}_{1}$ ( $\mathrm{r} 0.135, \mathrm{p}=0.03$ ) and arterial oxygen saturation ( $\mathrm{r} 0.161, \mathrm{p} 0.01$ ).

Conclusion COPD patients have evidence of premature ageing (shortened TL) compared to normal subjects irrespective of their smoking history. TL relates to $\mathrm{FEV}_{1}, \mathrm{SatO}_{2}$, exacerbation rate and hospitalisations.

The ECLIPSE study (GSK Study No. SCO104960, NCT00292552) was sponsored by GlaxoSmithKline.

\section{S50 AIRWAY SMOOTH MUSCLE INFLAMMATION IS CONTROLLED BY MICRORNA-145 TARGETING OF SMAD3 IN COPD}

L O'Leary, B Tildy, E Papazoglou, IM Adcock, KF Chung, MM Perry. Imperial College London, London, UK

\subsection{6/thoraxinl-2014-206260.56}

Introduction and objectives Airway smooth muscle cells (ASMCs) may contribute to the pathological airway inflammation and remodelling in COPD through the secretion of inflammatory cytokines and increased proliferation. Our previous work demonstrated that ASMCs from patients with COPD release greater amounts of IL-6 and CXCL8 compared to those from healthy subjects and are in a state of hyperproliferation. MicroRNAs (miRNAs) have recently emerged as important homeostatic regulatory molecules in COPD, and we have previously demonstrated the role of these in controlling ASMC proliferation in asthma. We hypothesise that microRNA-145 (miR-145) controls the aberrant phenotype observed in ASMCs from patients with COPD by targeting SMAD3, an important downstream signalling molecule of the TGF- $\beta$ pathway.

Methods Human primary ASMCs were grown from individuals classified as being healthy non-smokers, healthy smokers, or those with COPD ( $\mathrm{n}=9$ per group). Cells were stimulated with TGF- $\beta$ and foetal calf serum, and miRNA and mRNA expression levels were measured by RT-PCR. IL- 6 and CXCL8 release was measured by ELISA. Transfection of miR-145 mimics and inhibitors were used to model the effects of miR-145 over-expression and knock-down, respectively.

Results Low concentrations of TGF- $\beta$ significantly upregulated SMAD3 expression in ASMCs from patients with COPD. Higher concentrations of TGF- $\beta$ led to a suppression of SMAD3 expression, with a concomitant increase in miR-145 expression in these cells, to a greater degree than in healthy subjects.

Inhibiting miR-145 in ASMCs from COPD patients reduced the increased IL- 6 and CXCL8 release and proliferation back to levels comparable to that of healthy individuals.

Conclusions This is the first time that miR-145 has been demonstrated to be important in controlling the increased inflammatory state of ASM cells from COPD patients. This miRNA may not only act as a novel biomarker for COPD, but may also be a novel target for treatment.

\section{S51 CIRCULATING DESMOSINE RELATES TO CARDIOVASCULAR COMORBIDITY, CORONARY ARTERY CALCIFICATION SCORE (CACS), SYSTEMIC INFLAMMATION AND MORTALITY IN PATIENTS WITH COPD}

${ }^{1}$ Roberto A Rabinovich, ${ }^{2}$ Bruce E Miller, ${ }^{3}$ Karolina Wrobel, ${ }^{1}$ Gourab Choudhury, ${ }^{1}$ Kareshma Ranijt, ${ }^{1}$ Ellen M Drost, ${ }^{3}$ Lisa D Edwards, ${ }^{4}$ David A Lomas, ${ }^{5}$ Stephen I Rennard, ${ }^{6}$ Alvar Agusti, ${ }^{2}$ Ruth Tal-Singer, ${ }^{7}$ Jergen Vestbo, ${ }^{8}$ Emiel Wouters, ${ }^{9}$ Edwin Van Beek, ${ }^{10}$ John T Murchison, ${ }^{1}$ William MacNee, ${ }^{3}$ Jeffrey TJ Huang. ${ }^{1}$ Edinburgh Lung and the Environment Group Initiative (ELEGI), Centre for Inflammation and Research, Queens Medical Research Institute, Edinburgh, Edinburgh, UK; ${ }^{2}$ GlaxoSmithKline, King of Prussia, Pennsylvania, USA; ${ }^{3}$ Medical Research Institute, School of Medicine, University of Dundee, Dundee, UK; ${ }^{4}$ Faculty of Medical Sciences, University College London, London, UK; ${ }^{5} 5$ - Division of Pulmonary, Critical Care, Sleep and Allergy, University of Nebraska, Omaha, Nebraska, USA; ${ }^{6}$ Hospital Clinic, IDIBAPS, Universitat de Barcelona and CIBER Enfermedades Respiratorias, FISIB, Mallorca, Spain; 'Department of Respiratory Medicine, Odense University and University of Southern Denmark, Odense, Denmark; ${ }^{8}$ Department of Respiratory Medicine, Maastricht University Medical Centre, Maastricht, Maastricht, The Netherlands; ${ }^{9}$ Clinical Research Imaging Centre, Queens Medical Research Institute, Edinburgh, UK; ${ }^{10}$ Royal Infirmary of Edinburgh, Scotland, Edinburgh, UK

10.1136/thoraxjnl-2014-206260.57 


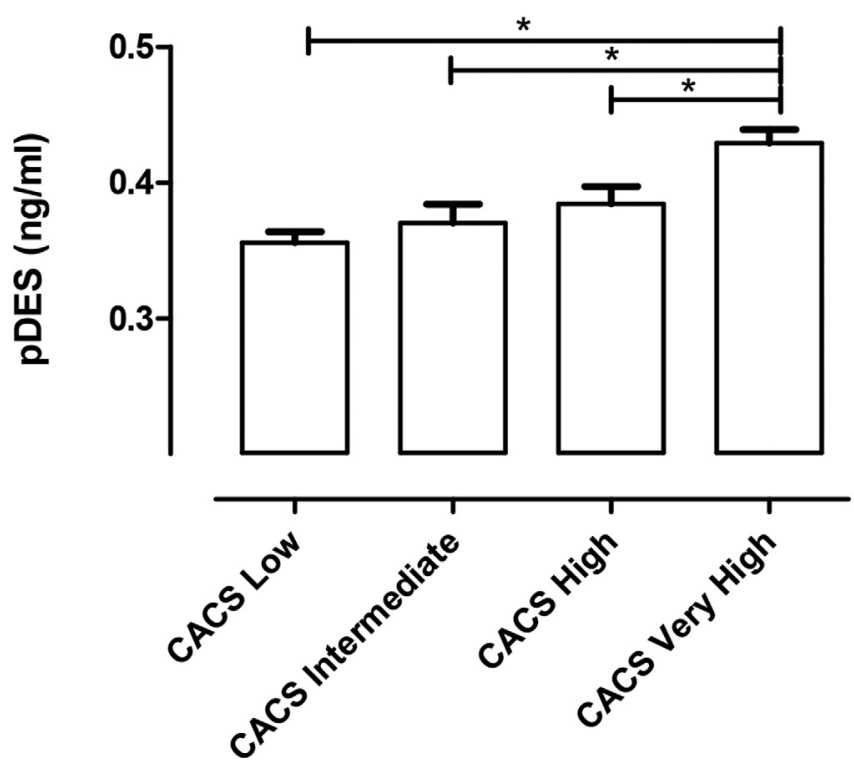

Abstract S51 Figure 1 Differences in pDES between patients with very high CACS and lower CACS levels ( ${ }^{*} p<0.01$ )

Introduction COPD is a risk factor for cardiovascular comorbidities. Elastin degradation represents a shared mechanism for the pulmonary and vascular features.

Methods and Results Plasma desmosine (pDES), a marker of elastin degradation, was measured in 955 COPD patients (609 male, age $63.1 \pm 7.2$ years, $\mathrm{FEV}_{1} 50.6 \pm 15.1 \%$ predicted) by an isotope dilution LC-MS/MS method. Coronary artery calcification (CACS), a surrogate of atherosclerosis, was assessed in 440 standard CT scan images (low $1000 \mathrm{AU}$ ).

Results pDES was elevated in patients with cardiovascular comorbidities $(\mathrm{p}<0.01)$ and correlated with $\mathrm{FEV}_{1}(\mathrm{r}=0.39, \mathrm{p}$ $<0.0001)$, MMRC $(\mathrm{r}=0.16, \mathrm{p}<0.0001), 6 \mathrm{MWD}(\mathrm{r}=-0.16, \mathrm{p}$ $<0.0001)$, BODE index $(\mathrm{r}=0.10, \mathrm{p}<0.005)$, fibrinogen, IL6, IL8, CCL18, and SPD but not with emphysema. These variables showed significant higher values in the patients in the highest pDES quartile. pDES was elevated in patients with very high CACS in comparison with patients with lower CACS (Figure 1) and in patients that died during a 3 year follow-up $(\mathrm{p}<0.0001)$. Conclusion pDES relates to lung function, systemic inflammation, cardiovascular comorbidities, and CACS in patients with COPD. pDES is a predictor of all cause overall mortality.

The ECLIPSE study (GSK Study No. SCO104960, NCT00292552) was sponsored by GlaxoSmithKline.

\section{How does clinical respiratory physiology help the clinician?}

\section{S52 IS A RAISED BICARBONATE, WITHOUT HYPERCAPNIA, PART OF THE PHYSIOLOGICAL SPECTRUM OF OBESITY- RELATED HYPOVENTILATION?}

${ }^{1}$ A Manuel, ${ }^{2} \mathrm{~N}$ Hart, ${ }^{1} \mathrm{~S}$ Stradling. 'Oxford Centre for Respiratory Medicine, Oxford Biomedical Research Centre, Churchill Campus, Oxford University Hospitals NHS Trust, OXford, OX3 7LI, London, UK; ${ }^{2}$ Respiratory and Critical Care Medicine, NIHR Comprehensive Biomedical Research Centre, London, UK

\subsection{6/thoraxjnl-2014-206260.58}

Introduction Obesity Hypoventilation Syndrome (OHS) is conventionally defined by the combination of obesity $\left(\mathrm{BMI}>30 \mathrm{~kg} / \mathrm{m}^{2}\right)$ and daytime hypercapnia $\left(\mathrm{PaCO}_{2}>6 \mathrm{kPa}\right.$, with no other explanation)

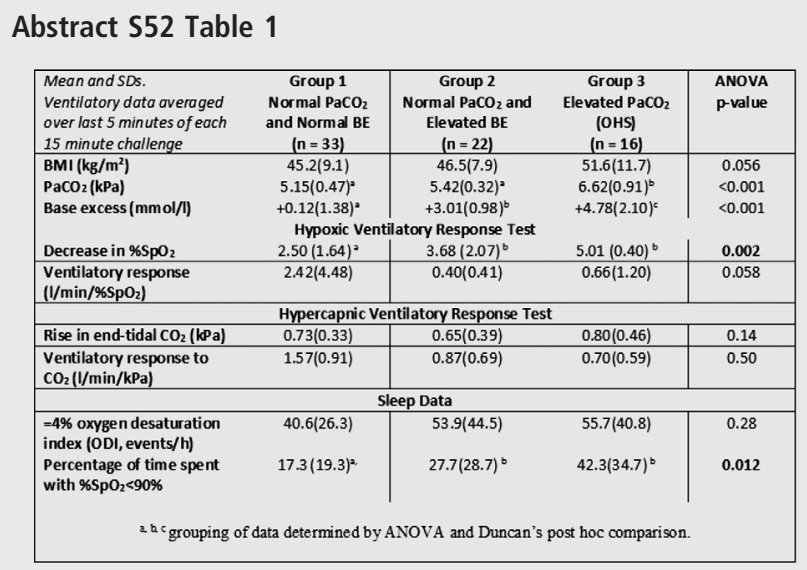

and sleep-disordered breathing may or may not be included. OHS patients have a higher morbidity, mortality, and health care utilisation compared with non-hypercapnic obese subjects. We hypothesised that in obese patients, even in the absence of a raised daytime $\mathrm{PaCO}_{2}$, the presence of a raised plasma standard bicarbonate, or base excess (BE, as a biomarker of whole body acid-base balance) would be associated with some well-recognised features of OHS (reduced ventilatory drives to hypoxia and hypercapnia, and nocturnal hypoventilation), thus suggesting they represent 'early' OHS.

Methods Obese subjects (BMI $>30 \mathrm{kgs} / \mathrm{m}^{2}$ ) were identified from a variety of sources, and divided into those with: 1) normal arterial blood gases and normal acid-base balance, 2) an isolated raised arterial $\mathrm{BE}(\geq 2 \mathrm{mmol} / \mathrm{L})$, and 3 ) awake arterial hypercapnia $(>6$ $\mathrm{kPa}$, i.e. established $\mathrm{OHS}$ ). Two-point ventilatory responses to hypoxia (15 min poikilocapnic response to $15 \% \mathrm{O}_{2}$ ) and hypercapnia (15 min response to $5 \% \mathrm{CO}_{2}$ in $\mathrm{O}_{2}$ ) were performed. Derivatives included the fall in $\mathrm{SaO}_{2}$ and rise in end-tidal $\mathrm{CO}_{2}$ when stable, and conventional ventilatory drive calculations. Polygraphic sleep studies were done with the derivatives of intermittent (oxygen desaturation index) and prolonged hypoxia (time below $90 \% \mathrm{SaO}_{2}$ ) reported here.

Results 71 subjects (BMI 47.2, SD 9.8; age 52.1, SD 8.8) were recruited into the above three groups $(33,22$, and 16 respectively). The table shows the $\mathrm{BMI}, \mathrm{PaCO}_{2}$ and $\mathrm{BE}$ for the three groups, along with the selected derivatives of the ventilatory drive measurements and sleep studies. For nearly all the ventilatory response and sleep study derivatives, group 2 (with only an isolated raised $\mathrm{BE}$ ) represented a middling group, and for some of the measures this middle group was more similar to group 3, with established OHS, rather than group 1.

Conclusion This study shows that obese individuals with raised BE, but without awake hypercapnia, probably represent an intermediary stage towards overt obesity-hypoventilation syndrome. Further studies will be required to establish if early intervention for this group is beneficial.

\section{S53 NEURAL RESPIRATORY DRIVE AND SYMPTOMS LIMITING EXERCISE CAPACITY IN CHRONIC OBSTRUCTIVE PULMONARY DISEASE}

${ }^{1} \mathrm{CJ}$ Jolley, ${ }^{2}{ }^{Y} \mathrm{M}$ Luo, ${ }^{3} \mathrm{~J}$ Steier, ${ }^{4} \mathrm{~K}$ Sylvester, ${ }^{5} \mathrm{~W}$ Man, ${ }^{1} \mathrm{G}$ Rafferty, ${ }^{5} \mathrm{MI}$ Polkey, ${ }^{1} \mathrm{~J}$ Moxham. ${ }^{1}$ King's College London, London, UK; ${ }^{2}$ Guangzhou Medical College, Guangzhou, China; ${ }^{3}$ Guy's and St Thomas' NHS Foundation Trust, London, UK; ${ }^{4}$ Addenbrookes Hospital, Cambridge, UK; ${ }^{5}$ Royal Brompton and Harefield NHS Foundation Trust, London, UK

10.1136/thoraxjnl-2014-206260.59 